\title{
A Metal-Ceramic Coaxial Cable Fabry-Pérot Microwave Interferometer for Monitoring Fluid Dielectric Constant
}

\author{
Shixuan Zeng ${ }^{\mathrm{a}}$, Adam Trontz ${ }^{\mathrm{a}}$, Wenge Zhu ${ }^{\mathrm{b}}$, Hai Xiao ${ }^{\mathrm{b}}$, Junhang Dong ${ }^{\mathrm{a}, 1}$ \\ ${ }^{a}$ Department of Biomedical, Chemical and Environmental Engineering, University of Cincinnati, \\ Cincinnati, Ohio 45221, USA \\ ${ }^{\mathrm{b}}$ Electrical and Computer Engineering Department, Clemson University, Clemson, SC 29634, USA
}

\begin{abstract}
A metal-ceramic coaxial cable Fabry-Pérot interferometer (MCCC-FPI) has been developed as a new microwave sensor and demonstrated for fast and reliably measuring and continuously monitoring dielectric constants for pure and mixed liquids. The sensor only requires a simple reference scan of the interferogram for the FPI with its sensing chamber filled with air or under vacuum to determine the actual inter-reflector length. The sensor is validated by measuring room temperature dielectric constant $\left(\varepsilon_{r}\right)$ at high frequencies around 1.4 - 4.7 GHz for three vegetable oils (corn, sesame, and olive), the synthetic Mobil $^{\circledR}-1$ engine oil, and mixtures containing sesame oil and water. The sensor has been also successfully demonstrated for determining $\varepsilon_{r}$ values for the Mobil-1 oil at various temperatures and for the welldispersed sesame oil-water mixtures of different compositions. The Bottcher's model of effective medium theory is shown to be better suited for predicting the $\varepsilon_{r}$ of the sesame oil-water mixture than the MaxwellGarnett and Bruggeman equations of spherical solutions.
\end{abstract}

Keywords: metal-ceramic coaxial cable; Fabry-Pérot interferometer; dielectric constant, fluid

\footnotetext{
${ }^{1}$ Corresponding author at: Department of Biomedical, Chemical and Environmental Engineering, University of Cincinnati, Cincinnati, Ohio 45221, USA.

E-mail address: Junhang.dong@uc.edu (J. Dong).
} 


\section{Introduction}

The knowledge of fluid dielectric properties is needed in the design and operation of electrical power transmission equipment, electronic devices, microwave communication networks, and other high frequency technologies such as microwave chemical synthesis, biological materials analysis, and food processing, etc. Thus, continued efforts have been made in developing and improving technologies for measuring gas and liquid dielectric constants to serve specific applications. In the past few decades, different microwave techniques have been established for studying fluid dielectric behaviors to meet the needs in many fast-developing technologies such as radar detection, wireless communication, microwave synthesis, and biological tests, etc. On the other hand, the understandings of relationships between the fluid dielectric constant and its chemical, physical, and thermodynamic properties allow developing new paradigms of sensors with the capability of detecting changes in fluid chemical composition and thermodynamic state using microwave signals.

A number of microwave techniques have been reported for fluid dielectric constant measurements, including the short-circuit coaxial probing, waveguide or coaxial transmission-line sensing, resonant cavity perturbation detection, and admittance cell equivalent thickness analysis, etc. [1-4] Operations of these dielectric sensing devices rely on the generation and analyses of microwave signals including time domain spectra and frequency domain spectra, and involve relatively simple instrumentations. However, these sensors often encounter various issues, such as errors from poor contacts, intermittent operations, repeating calibrations, complicated sample preparation, and challenges in precision fabrication of probing devices. [5] Here, we demonstrate a novel metal-ceramic coaxial cable (MCCC) Fabry-Pérot interferometer (FPI) as microwave sensing device for measuring and continuous monitoring of fluid dielectric constant.

Coaxial cable is a microwave transmission line constituted by an inner conductor coaxially centered in a tubular outer conductor with the cylindrical space between them filled by a dielectric material (i.e. electrical insulator). The structure of the coaxial cable FPI (CC-FPI) is schematically illustrated in Figure 
1. The microwave FPI is established by two dielectric perturbations, i.e. the two small-size inserts with a different dielectric constant $\left(\varepsilon_{r, 2}\right)$ placed in the original insulator $\left(\varepsilon_{r, 1}\right)$ in a distance of " $d$ ", which act as weak reflectors in the coaxial transmission line. The incident microwave propagating along the coaxial cable generates two reflections $\left(\mathrm{U}_{1}\right.$ and $\left.\mathrm{U}_{2}\right)$ at the two reflectors. The two reflections, $\mathrm{U} 1$ and $\mathrm{U} 2$ as expressed by equations (1) and (2), respectively, form an interferogram traveling backwards. The intensity of the reflected interfering microwave signal (U) can thus be expressed by equation (3).

$$
\begin{aligned}
& U 1=\Gamma(f) e^{-\alpha z} e^{-j 2 \pi f \frac{2 d_{0} \sqrt{\varepsilon_{r, 1}}}{c}} \\
& U 2=-\Gamma(f) e^{-\alpha z} e^{-j 2 \pi f \frac{2 d_{0} \sqrt{\varepsilon_{r, 1}}+2 d \sqrt{\varepsilon_{r}}}{c}} \\
& U=2 j \cdot \Gamma(f) e^{-\alpha z} e^{-j 2 \pi f \frac{2 d_{0} \sqrt{\varepsilon_{r, 1}}+d \sqrt{\varepsilon_{r}}}{c}} \sin \left(2 \pi f \frac{d \sqrt{\varepsilon_{r}}}{c}\right)
\end{aligned}
$$

where $\Gamma(f)$ is the reflection coefficient at the reflectors, as a function of microwave frequency $f ; \alpha$ is the propagation attenuation coefficient; $z$ signifies the propagation direction; $\mathrm{d}_{0}$ is the propagation distance prior to the first reflector; $\varepsilon_{r}$ is the relative permittivity of the insulation between the reflectors; $c$ is the speed of light in vacuum; and $d$ is the inter-reflector distance. Thus, the reflected interferogram can be recorded to monitor changes in the $d \sqrt{\varepsilon_{r}}$ value for the inter-reflector segment.

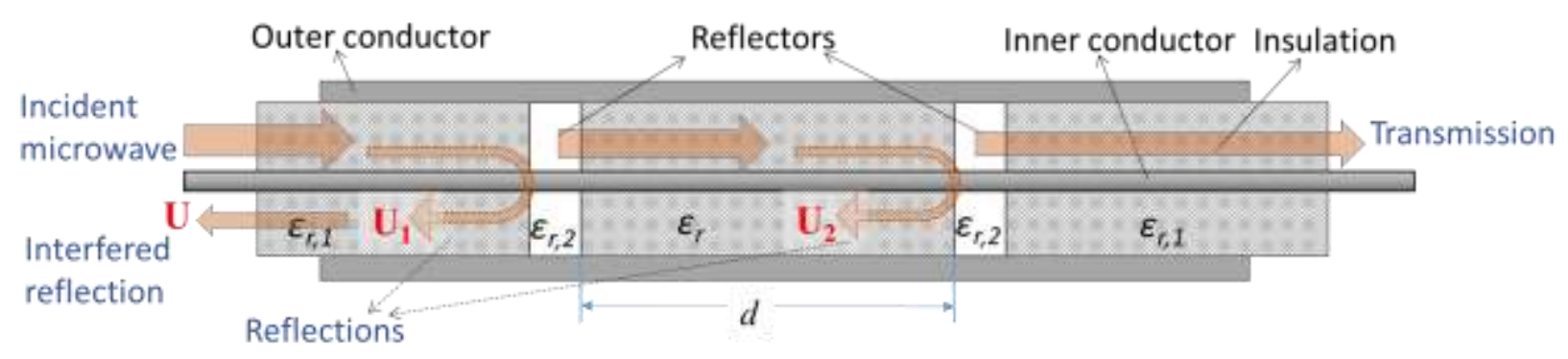

Figure 1. Schematic showing the structure of the coaxial cable Fabry-Pérot microwave interferometer.

The concept of CC-FPI was first demonstrated and applied to detecting large strain and measuring low temperature using commercially available radio frequency communication coaxial cables. [6-8] These FPI sensors were constructed by simply drilling periodical holes through the outer conductor and plastic insulation layer to serve as dielectric perturbations. Such simple CC-FPI sensors showed the capability of 
measuring strain and low temperature through monitoring the interferometric signal $(U)$, which as expressed by equation (3) shifts upon changes of $d$ and/or $\mathcal{E}_{r}$ induced by strain and/or temperature variations. [6-7] More recently, we developed a new type of metal-ceramic coaxial cable FPI (MCCCFPI) sensor for measurement of high temperature (up to $1000^{\circ} \mathrm{C}$ ). [9] The MCCC-FPI consisted of metal tube and wire as outer and inner conductors, respectively, and ceramic tubes as insulators where a pair of small-width air gaps was created to serve as reflectors. In these reported CC-FPI sensors, the interreflector insulation segments were of the same materials as those in their respective original cables for convenience, and hence $\varepsilon_{r}=\varepsilon_{r, l}$. The inter-reflector segment of insulation may be replaced by different dielectric materials $\left(\varepsilon_{r} \neq \varepsilon_{r, 1}\right)$ to offer broader opportunities for achieving special sensing abilities.

\section{Sensor concept}

In the new MCCC-FPI sensor of this work, the inter-reflector segment of insulation material in the coaxial cable is removed to leave a free space between the conductors. This inter-reflector space serves as the sensing chamber allowing fluid sample to fill in or continuously flow through as schematically depicted in Figure 2. This MCCC-FPI sensor can thus monitor the interferogram phase shift caused by changes in dielectric constant of the fluid $\left(\varepsilon_{r}\right)$ filling the chamber when the chamber length $(d)$ is fixed. The length $d$ is a constant when operating at room temperature and can be readily calibrated using the coefficient of thermal expansion (CTE) of the metal conductor material when involving temperature variations. 


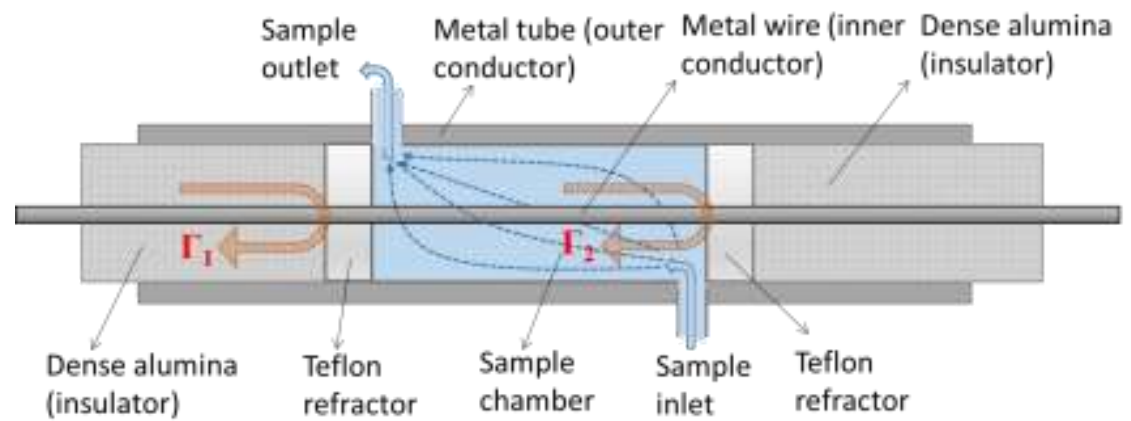

Figure 2. Schematic diagram of the MCCC-FPI sensor and microwave reflection.

The interferogram phase shift caused by the time delay of the reflection from the second reflector is a function of " $d \sqrt{\varepsilon_{r}}$ " in a given MCCC-FPI. Therefore, when the chamber length $d$ is determined, the change in fluid $\varepsilon_{r}$ can be obtained from phase shift of the interferogram in frequency domain. In sensing operation, the frequency of the $\mathrm{N}^{\text {th }}$ resonant peak $\left(f_{N}\right)$ is tracked and its relation with the time delay $\left(\tau=2 \mathrm{~d} \sqrt{\varepsilon_{r}} / c\right)$ is deduced from equation (3) as

$$
f_{N}=\frac{N c}{2 d \sqrt{\varepsilon_{r}}}
$$

Thus the dielectric constant $\varepsilon_{r}$ of the fluid in the sensing chamber can be determined through monitoring the $f_{N}$ value. The thus determined $\varepsilon_{\mathrm{r}}$ values of the fluid sample are corresponding to the respective resonance frequencies $\left(f_{N}\right)$ being used in equation (4). Taking advantage of the ability of current VNA in ultra-fast scanning interfering spectrum, continuous and real-time online monitoring of dielectric constant of fluid flow can be readily accomplished. Also, the chamber length $d$ can be accurately determined using the $f_{N}$ value obtained under vacuum or dry air flow $\left(\varepsilon_{r} \sim 1.0\right)$ that avoids the need of precision manufacturing and the uncertainty in structural changes overtime.

\section{Sensor fabrication}


The following materials were used for constructing the MCCC-FPI sensor. The stainless steel (SS) tube (5.4 mm outer diameter and $3.1 \mathrm{~mm}$ inner diameter; grade 316, ASTM A213/A269, McMaster Carr) and SS wire (diameter of $1.34 \mathrm{~mm}$; grade 316L, ASTM A555/A580, McMaster Carr) were used as outer and inner conductors, respectively. The electric conductivity and CTE of the SS material are $11.6 \mu \Omega / \mathrm{cm}$ and $8.9-11.1 \times 10^{-6}{ }^{\circ} \mathrm{C}^{-1}$, respectively. Dense open-ended extruded alumina tubes $(3.05 \mathrm{~mm}$ outer diameter and $1.35 \mathrm{~mm}$ inner diameter, $99.5 \% \alpha-\mathrm{Al}_{2} \mathrm{O}_{3}, 95 \%$ density, Ceramic Solutions Inc.) were used as the dielectric insulator. The alumina adhesive (Ceramabond 503, $\alpha$-alumina, AREMCO Inc.) was used to firmly attach the alumina tube insulator to the inner conductor. The outer surface of the alumina tubes were polished to ease the difficulty in assembling the rigid parts in the tightly fitted MCCC. The dielectric constant and CTE of the alumina tube are $9.5-12.0$ and $8.4 \times 10^{-6}{ }^{\circ} \mathrm{C}^{-1}$, respectively. The two reflectors are made of Teflon discs with a thickness of $3 \mathrm{~mm}$. The dielectric constant of the Teflon disc is $\sim 2.0$ at room temperature. The Teflon discs were made to tightly fit with the tube and wire so that fluid leaking into the minimal gaps between the alumina tube and conductors is prevented. Solvent-resistant silicone sealant may be applied to the reflector fittings for ensuring the tightness of seal. The commercially obtained sesame oil (Cookwell, Sovena USA), olive oil (Great Value, Bentonville, USA), corn oil (Great Value, Bentonville USA), Mobil-1 engine oil (ExxonMobil Oil Corp.) were used as sample fluids. The oils were dehydrated by zeolite adsorbents (96096-100G, Sigma-Aldrich) prior to measurements. These specific fluids were selected in this work for proof of concept and demonstration of the sensor operation for fluid dielectric constant measurements mainly because of safety and environmental control requirements in the optoelectronic labs.

The photographs in Figure 3 show the assembly of conductors (SS tube and wire), alumina tube insulators, Teflon reflectors, and the sample chamber in the actual MCCC-FPI sensor. At the two ends of the sample chamber in the cable, two 3.1-mm-diameter holes were drilled through the SS tube outer conductor on opposite sides of the tube diameter as entrance and exit for the fluid flow. Transparent Teflon tubes of 3.1-mm in outer diameter were tightly fitted into these two holes as fluid piplines, which 
were connected to a tube pump. The two 3-mm-thick Teflon disks were glued onto the alumina tube ends in the chamber using slicon sealant or epoxy. The distance between the two Teflon disc reflectors was roughly controlled around $10 \mathrm{~cm}$ during fabrication and precisely determined by interferometric measurement after the sensor was assembled. The assmbly was cured at room temperature for 24 hours and the sealing effect around the chamber was confrimed by checking the nitrogen gas or water leakage at the open ends of the MCCC. The sensing chamber was then cleaned by circulating deionized water for over $100 \mathrm{~h}$ and then fully dried in an vacuume oven at $50^{\circ} \mathrm{C}$. The long-time water circulation was also used to re-test the sealing effects of the chamber before sensing operation.

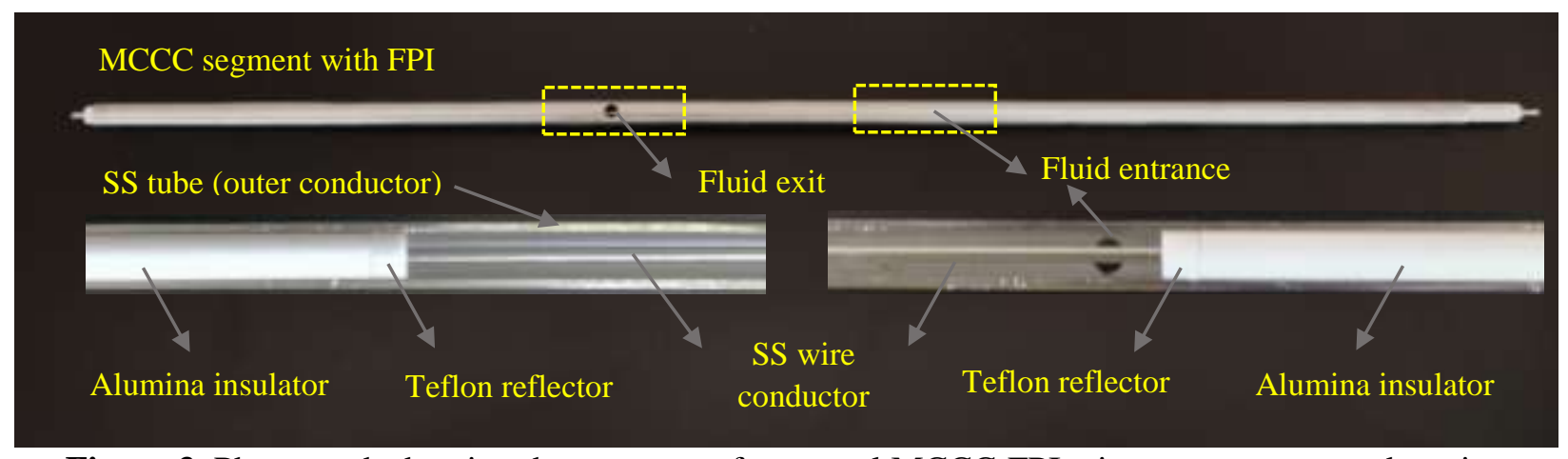

Figure 3. Photograph showing the structure of an actual MCCC-FPI microwave sensor and section revealing the assembly of alumina insulators, Teflon disc reflectors, SS wire inner conductor, and fluid sample chamber.

\section{Apparatus and operation}

The experimental appratus used for operating the MCCC-FPI sensor for measuring fluid dielectric constant is schematically illustrated in Figure 4. One end of the MCCC-FPI was connected through a communicate coaxial cable to a vector network analyzer (N9923A RF VNA, Keysight Technologies) and the other end was free-end with short-circuit-termination. The sample fluid, which was stored in a glass flask, was circulated through the sensing chamber by a digital tube pump (Masterflex, Model 77202-50). The MCCC-FPI together with the flask reservoir and tubing was submerged in a water bath $\left( \pm 0.1^{\circ} \mathrm{C}\right.$; 
Branson 3510) for experimentally studying the effects of temperature. The fluid flow entered the sensing chamber from the bottom side hole and exited from the hole on the top and the liquid flow was verified to be free of bubbles during pumping circulation. The the microwave spectrum was swept by the VNA at a step of $6 \mathrm{MHz}$ over a band width range from $0.1 \mathrm{GHz}$ to $6 \mathrm{GHz}$ and the data acquisition and processing were accomplished by a PC workstation connected to the VNA. The time needed for scanning an entire spectrum in the frequency range of $0.1-6 \mathrm{GHz}$ was $957 \mathrm{~ms}$. Thus a nearly real time monitoring capability can be achieved for the flowing fluid. The microwave signal was firstly processed by Fourier transform and then gated on the two reflection peaks to eliminate noises such as the reflections from the connections betweent the MCCC and communication cable and the short-circuit end. [10]

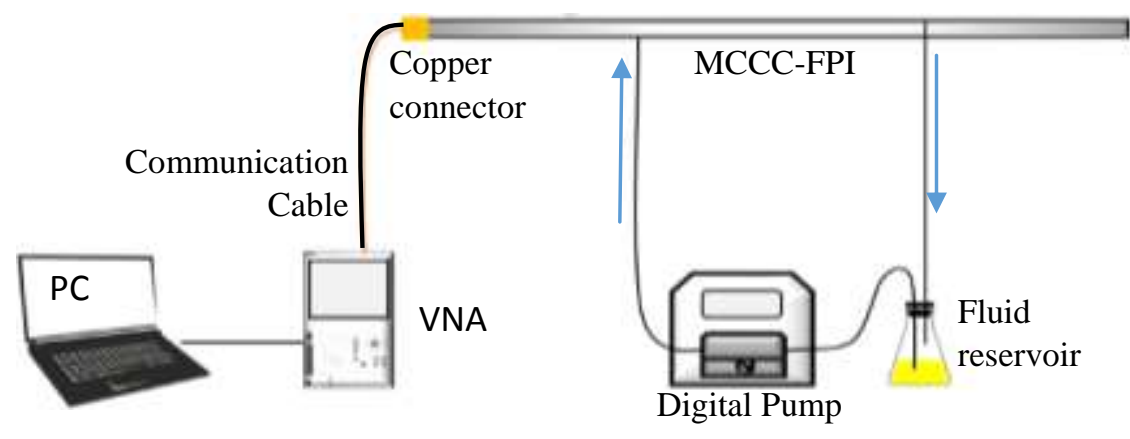

Figure 4. Schematic diagram of the MCCC-FPI sensor operation system.

The MCCC-FPI was firstly examined at room temperature with dry air $\left(\varepsilon_{r} \sim 1.00059\right)$ filling the chamber to test the microwave interferometric response of the sensor and to accurately determine the value of $d$. The sensor was then operated at room temperature for determining the dielectric constants of sesame oil, Mobil-1 oil, corn oil and olive oil, respectively, which were used to validate the FPI measurement method. During the experiment, the liquid sample was circulated for $30 \mathrm{~min}$ to ensure that chamber was fully filled without air bubble in the liquid flow prior to recording the interferometric spectra. The measurement of each sample oil was repeated for three times to ensure data reproduceability and an average of the three measurements was taken. 
Understanding the influence of temperature on the dielectric constant measurement is essential to practical applications. The causes of temperature-induced variations of the sensor's spectroscopic output mainly include the thermal expansion or contraction of the chamber length and the temperature-dependent fluid dielectric constant. The determination of temperature effect on fluid dielectric constant is a desired capability while the effect of thermal expansion or contraction of the chamber length on the $\varepsilon_{r}$ measurement is an error source, which must be kept neligible or readily calibrated. The actual value of $d$ at temperature $\mathrm{T}\left(d_{T}\right)$ can be obtained by:

$$
d_{T}=d_{R T}+d_{R T}(T-R T) \cdot C T E
$$

where $d_{R T}$ is the chamber length $\left(d_{R T}=9.75 \mathrm{~cm}\right)$ measured at room temperature $\left(21.6^{\circ} \mathrm{C}\right.$ in this study $)$ and CTE of the SS conductors can be considered as constant $\left(\sim 10^{-5}{ }^{\circ} \mathrm{C}^{-1}\right)$ within the small range of temperature variation involved. The MCCC-FPI was examined at temperatures varying from 21.6 to $50^{\circ} \mathrm{C}$ with the chamber filled with air to investigate the magnitude of thermal expansion effect on the dielectric constant measurements as represented by the absoulte deviation in $\varepsilon_{r}\left(\Delta \varepsilon_{r}\right)$ as defined by equation (6),

$$
\Delta \varepsilon_{r}=\left(\frac{N c}{2 d f_{N}}\right)^{2}-\left(\frac{N c}{2 d_{T} f_{N}}\right)^{2}
$$

With the understanding of the influence of MCCC thermal expansion on the $\varepsilon_{r}$ measurement, the MCCC-FPI was tested for analyzing the temperature-dependence of $\varepsilon_{r}$ for the oil samples. The frequency measurement was performed both ways of temperature increasing and decreasing and the range of error against the average value is analyzed for evaluating possible sensor structure change under temperature swing.

The ability of the MCCC-FPI sensor for continuously monitoring fluid dielectric constant was demonstrated at room temperature using sesame oil as a sample fluid when water was incrementally added to change the dielectric property of the constantly circulated oil flow. The experiment started with $30 \mathrm{ml}$ sesame oil pre-dehydrated in vacuum in a sealed 125-ml flask and deionized water was injected into the oil in flask by a precision volumetric syringe $( \pm 0.01 \mathrm{ml})$ under magnetic stirring. For each level of 
water content, a 5-min stirring-circulation was allowed before scanning the interferogram and another interferogram was taken after 15-min of further stirring-circulation. The immiscible oil-water mixture was well dispersed to exhibit uniformly opaque appearance, which was stable for more than an hour without forming appreciable droplets even under static condition.

\section{Results and discussion}

Figure 5 shows the time domain interferometric spectrum of the MCCC-FPI with air-filled chamber. The time delay between the two reflection peaks was $0.65 \mathrm{~ns}$ that gives a $\mathrm{d}$ value of $9.75 \mathrm{~cm}$ by equation (4), which is consistent with that measured in sensor construction $(\sim 9.7 \mathrm{~cm})$.

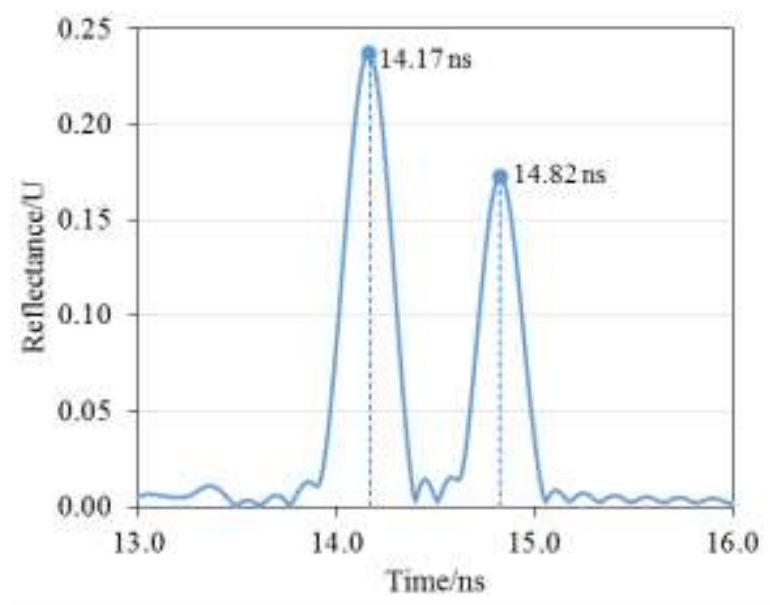

Figure 5. The time domain spectrum of the air-filled MCCC-FPI.

The interferograms for the four oil samples are presented in Figure 6, which show well-defined sharp resonant peaks that are desirable for reliable identification of resonance frequencies for $\varepsilon_{r}$ calculations. 


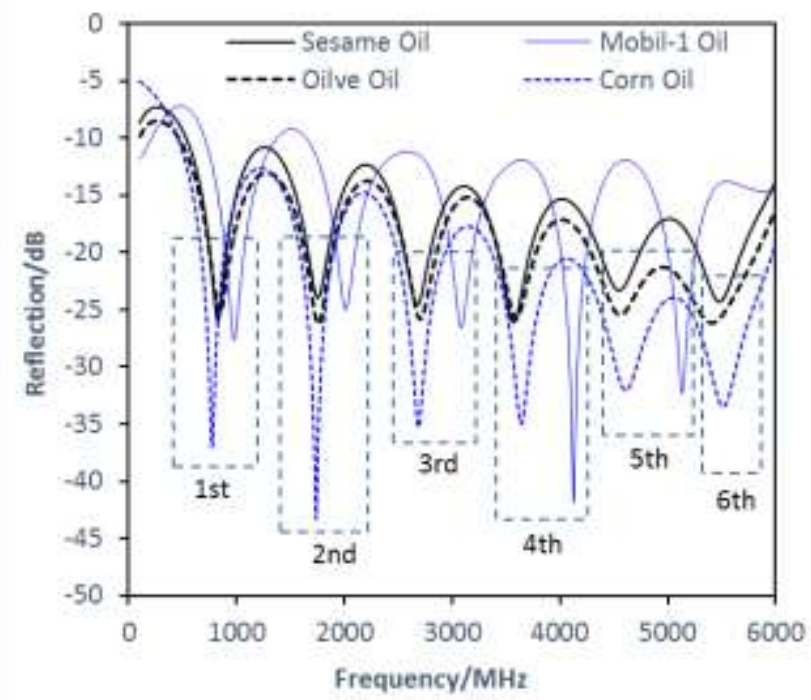

Figure 6. The frequency domain interferometric reflection spectra of the MCCC-FPI when filled with sesame oil, olive oil, corn oil, and Mobil-1 oil, respectively.

The fluid $\varepsilon_{\mathrm{r}}$ values corresponding to all the resonant peak frequencies observed in the experimental frequency range were calculated by equation (4) using the pre-determined chamber length $\mathrm{d}(=9.75 \mathrm{~cm})$ except for the first and last resonant peaks of which the data quality could be affected by the edge effects and gating operation in time domain. The results are summarized in Table 1 . The $3^{\text {rd }}$ resonance peak frequencies $\left(f_{3}\right)$ were used for calculating the fluid dielectric constants $\varepsilon_{r}$ in the rest of the experiments for demonstration. The $\varepsilon_{r}$ of the oils corresponding to the resonance frequencies measured by this new MCCC-FPI sensor agree well with the literature values. [11-16] The $\varepsilon_{r}$ values of the vegetable oils are significantly higher than that of the Mobil-1 oil because the former molecules contain hydroxyl groups and acid terminals to give much greater polarity than the latter, which is a nonpolar synthetic substitute of petroleum oil. The observed trend of slight decreases in $\varepsilon_{r}$ of the oils with the increasing frequency is also consistent with literature reports and indication of the Debye equations. [17-18] The small disagreement with the literature $\varepsilon_{r}$ values may be caused by minor differences in the complex compositions of the oils. As predicted by equation (4), the interferometric spectrum exhibited a red shift, i.e. shifting towards lower frequency, when $\varepsilon_{r}$ of the sample fluid in the chamber increased. In principle, the sensitivity of $\varepsilon_{r}$ 
measurement by the FPI sensor is depdendent of the chamber length $d$ and the frequency of the resonant peak being used and the measurement resolution is also depending on the step size used in spectrum scanning. The responding $f_{N}$ is propotional to $\left(\varepsilon_{r}^{-0.5}\right)$ with a propotionality of $(N c / 2 d)$, which, for the $3^{\text {rd }}$ resonant peak $(\mathrm{N}=3)$ and the current chamber length $d=9.75 \mathrm{~cm}$, is $4.615 \mathrm{GHz}$ to provide a sensitivity of $-1.79 \times 10^{-3} \mathrm{MHz}^{-1}$ when a linear approximation is made for the small range of $\varepsilon_{r}$ involved in this work as shown in Figure 7. Thus, for the scanning step of $6 \mathrm{MHz}$, the measurement resolution for $\varepsilon_{r}$ is 0.01 without spectrum curve fitting. However, there are off the shelf VNA units which can easily achieve scanning step well below $1 \mathrm{MHz}$ to obtain much finer $\varepsilon_{r}$ measurement resolution if needed.

Table 1. Dielectric constants of the oils measured at room temperature $\left(24^{\circ} \mathrm{C}\right)$ by the MCCC-FPI in comparison with literature values

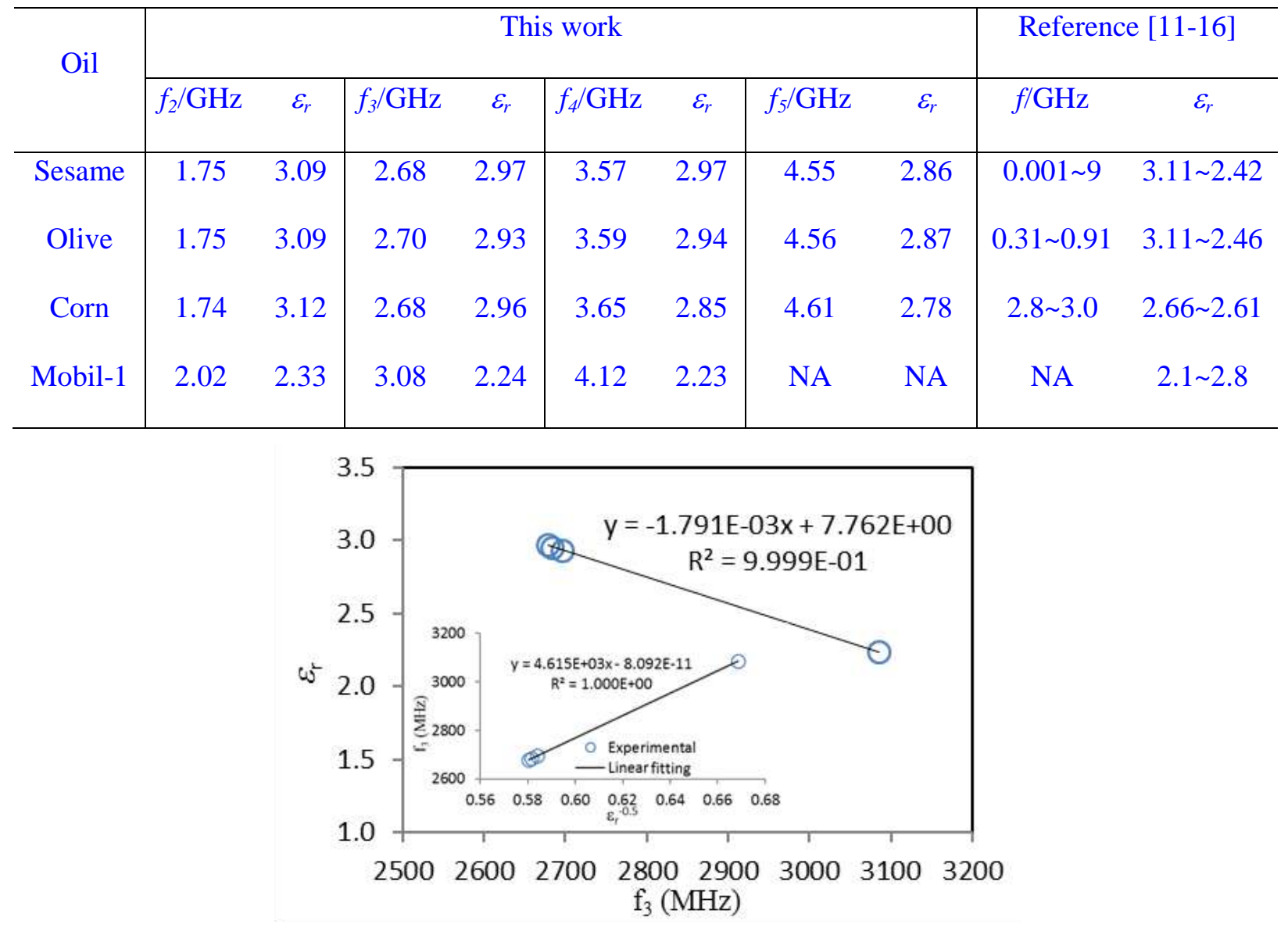


Figure 7. The relationship between the fluid $\varepsilon_{r}$ and the $3^{\text {rd }}$ resonant peak frequency for the MCCC-FPI (insert showing dependence of $f_{3}$ on $\varepsilon_{r}^{-0.5}$ ).

Figure 8 presents the experimentally measured frequency of the $2^{\text {nd }}$ resonance frequency $\left(f_{2}\right)$ of the air-filled MCCC-FPI and the corresponding $\Delta \varepsilon_{r}$ as a function of temperature. Since the change of $\varepsilon_{r}$ for the dry air is negligible in this temperature range $\left(21.6-50^{\circ} \mathrm{C}\right)$, the $\Delta \varepsilon_{r}$ represents error caused by uncalibrated $d$ value. The maximum $\Delta \varepsilon_{r}$ was less than $6 \times 10^{-4}$, which is far smaller than the $\varepsilon_{r}$ measurement resolution $\left(10^{-2}\right)$ determined by microwave scanning step. Thus, temperature calibration is not used here in analyzing the temperature-dependencies of $\varepsilon_{r}$ for the oils.

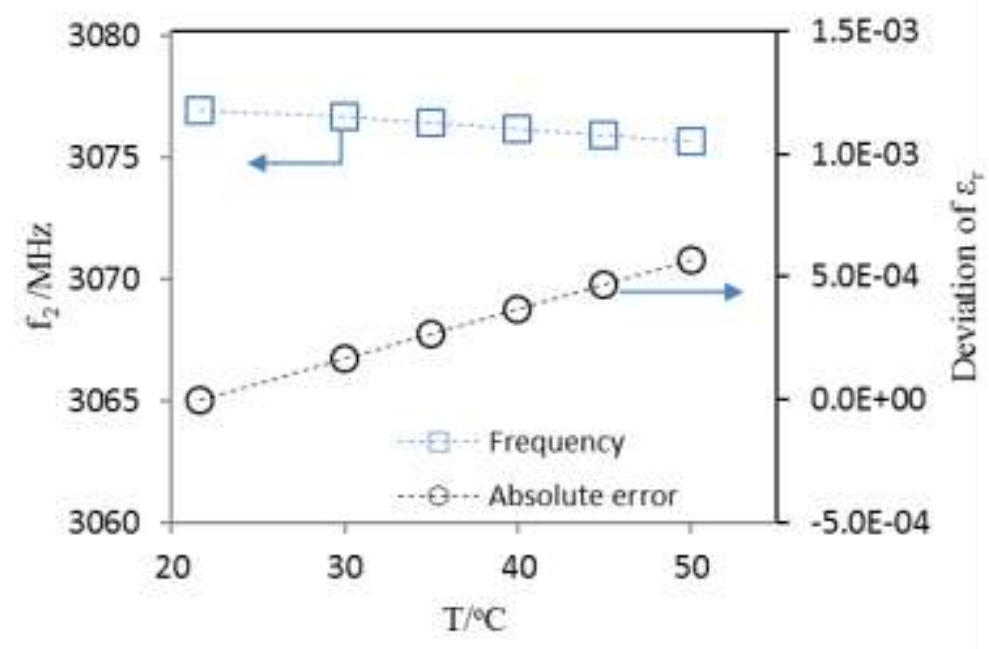

Figure 8. The $2^{\text {nd }}$ resonant peak frequency $\left(f_{2}\right)$ of dry air filled MCCC-FPI and the $\Delta \varepsilon_{r}$ as functions of temperature.

The Mobil-1 oil was used to demonstrate the measurement of temperature-dependence of its dielectric constant at $f_{3}$. The results are presented in Figure 9. The relative deviations between $\varepsilon_{r}$ values measured during heating and cooling processes was only $0.08 \%$, indicating that the sensor assembly is stable enough for operating with excellent consistency under temperature fluctuations and without calibrations. The $\varepsilon_{r}$ of the Mobil-1 oil displayed the anticipated typical temperature-dependency that is to decrease as 
temperature increases because the molecular kinetic energy is enhanced at higher temperature to prevent the alignment of weak dipoles along the electric field. [19]

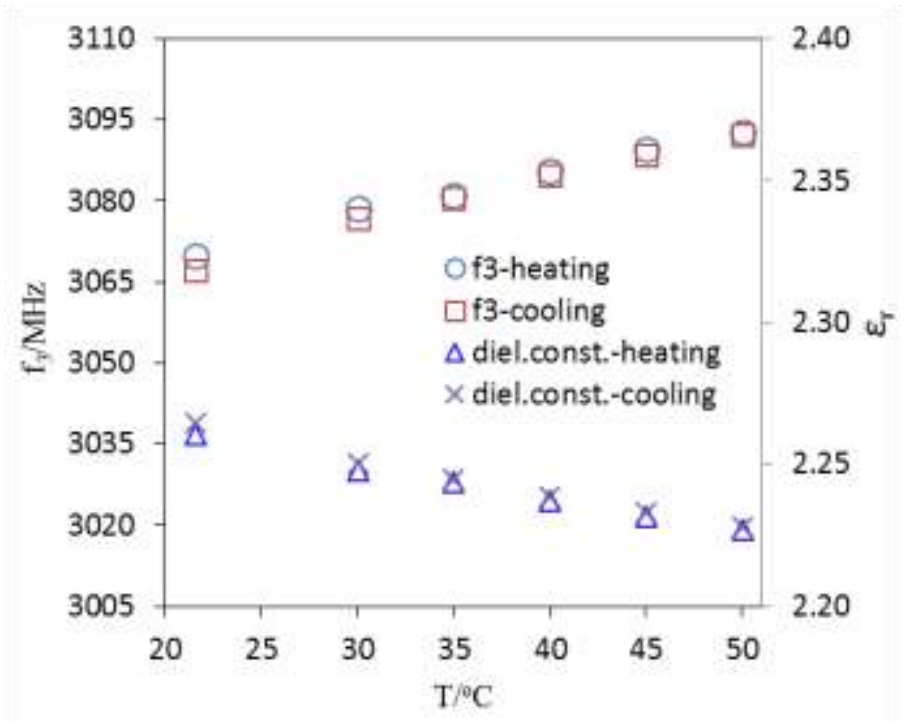

Figure 9. The $3^{\text {rd }}$ resonant peak frequency $\left(f_{3}\right)$ and dielectric constant $\varepsilon_{r}$ as functions of temperature for Mobil-1 engine oil.

The online monitored frequency domain spectra for the sesame oil flow are displayed as a function of oil weight percentage in Figure 10 (a) and the corresponding staircase showing of the frequency $\left(f_{3}\right)$ along the operation time is presented in Figure 10 (b). It should be noted that the time intervals between measurement points are for ensuring the mixing and homogenizing of the oil-water mixture in the entire flow system including the sensing chamber but not limited by the speed of spectrum canning and acquisition, which can be as fast as less than 1 second per scan of the spectrum. The measurement for online continuous monitoring can be much fast if the microwave frequencies to be scanned is limited to a necessary but much narrower range. 


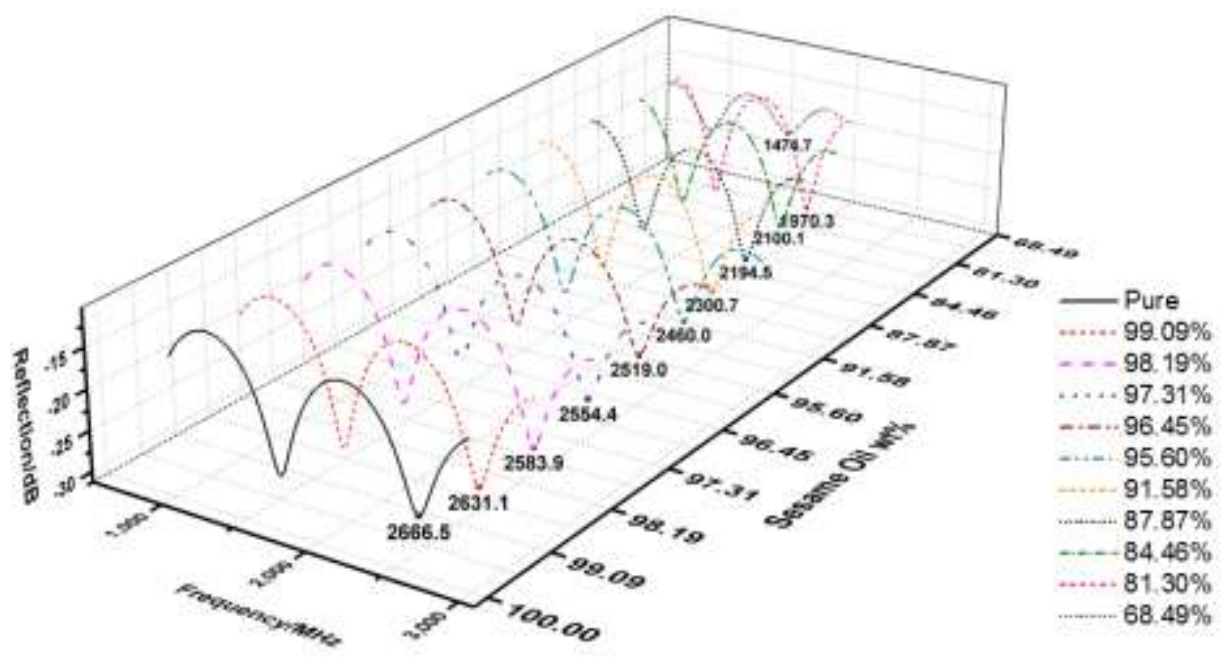

(a)

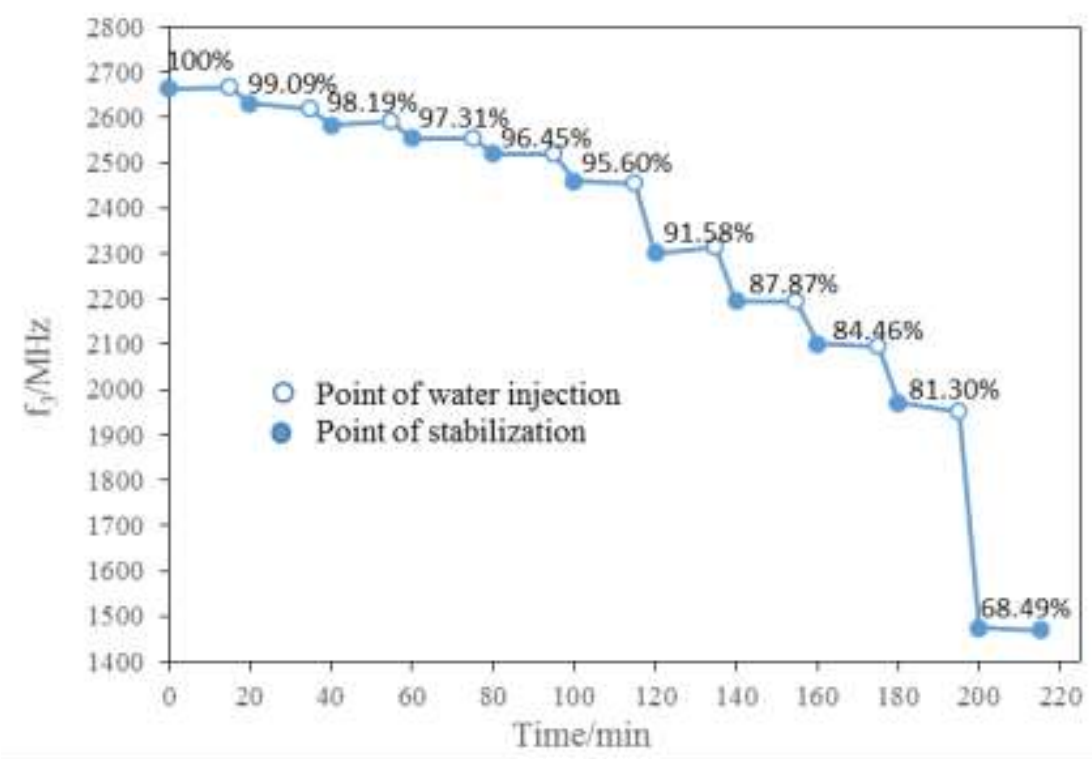

(b)

Figure 10. Results of online monitoring by the MCCC-FPI for sesame oil-water mixtures. (a) interferogram as a function of oil weight percent, and (b) $f_{3}$ as a function of operation time.

The online monitored $3^{\text {rd }}$ resonance $\left(f_{3}\right)$ and its corresponding $\varepsilon_{r}$ values are presented as functions of sesame oil weight percentage in Figure 11. As expected, dielectric constant of the mixture increased nonlinearly with increasing the water content because $\varepsilon_{r}$ of the water $\left(\varepsilon_{r} \sim 80.2\right)$ is far greater than that of 
the sesame oil $\left(\varepsilon_{r} \sim 2.97\right)$. The experimental data $\left(\varepsilon_{r . \exp }\right)$ are also compared with the predictions given by the Maxwell-Garnett (M-G) relation for spherical solutions, the Burggeman extended M-G for highly dispersed liquid mixtures, and the Bottcher model of effective medium, which are expressed by equations (7-9). [20-22]

$$
\begin{array}{ll}
\text { Maxwell-Garnett: } & \frac{\varepsilon_{r, \text { mix }}-\varepsilon_{r, s}}{\varepsilon_{r, m i x}+2 \varepsilon_{r, s}}=\frac{\varepsilon_{r, d}-\varepsilon_{r, s}}{\varepsilon_{r, d}+2 \varepsilon_{r, s}} \varphi_{d} \\
\text { Bruggeman: } & \frac{\varepsilon_{r, \text { mix }}-\varepsilon_{r, d}}{\varepsilon_{r, s}-\varepsilon_{r, d}}\left(\frac{\varepsilon_{r, s}}{\varepsilon_{r, m i x}}\right)^{1 / 3}=1-\varphi_{d} \\
\text { Bottcher: } & \frac{\varepsilon_{r, \text { mix }}-\varepsilon_{r, s}}{3 \varepsilon_{r, \text { mix }}}=\frac{\varepsilon_{r, d}-\varepsilon_{r, s}}{\varepsilon_{r, d}+2 \varepsilon_{r, m i x}} \varphi_{d}
\end{array}
$$

In the model calculations of $\varepsilon_{\mathrm{r}}$ for the sesame oil-water mixture, constant $\varepsilon_{\mathrm{r}}$ values were used for pure water and sesame oil because they are nearly unchanged in the small frequency range of $1.47-2.66 \mathrm{GHz}$ for the $3^{\text {rd }}$ resonance peak used. The relative errors of the predictions, which are defined as percentage deviations of the predicted $\varepsilon_{r, \text { mix }}$ from the experimental values $\left(\operatorname{error} \%=\left|\varepsilon_{r, \operatorname{mix}}-\varepsilon_{r, \exp }\right| / \varepsilon_{r, \exp }\right)$, were $9.0 \%, 7.0 \%$ and $3.0 \%$ for the $\mathrm{M}-\mathrm{G}$, Burggeman, and Bottcher models, respectively. The comparisons in average error and dependency of $\varepsilon_{r, m i x}$ on mixture composition shown in Figure 11 indicate that the effective medium theory based Bottcher equation is better suited than the M-G and its modified Bruggeman models for the highly asymmetric sesame oil-water mixtures.

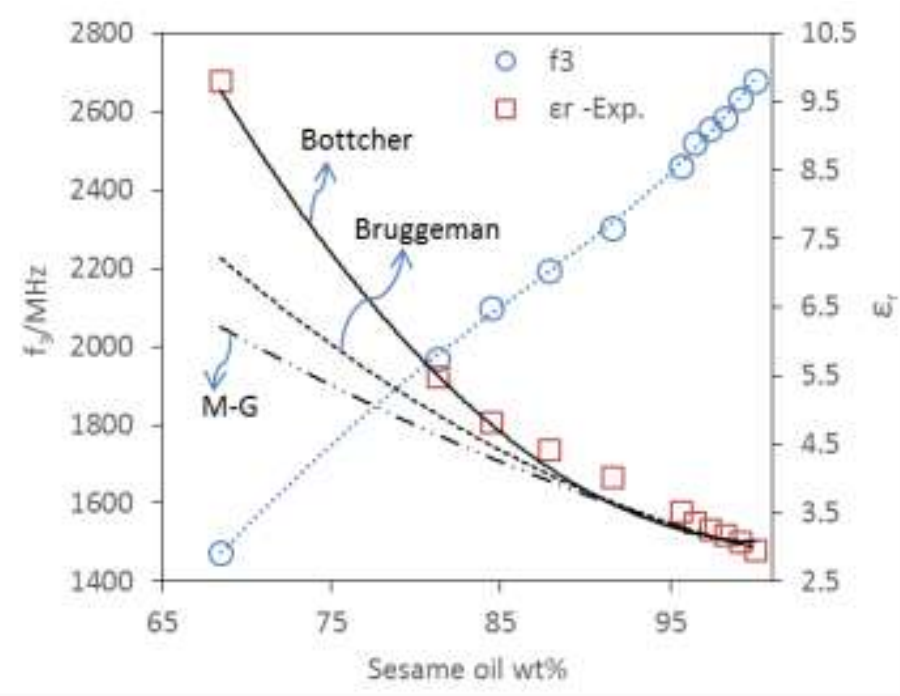


Figure 11. Experimentally determined dielectric constant of the sesame oil-water mixture as a function of weight percent of oil in comparison with model predictions.

\section{Conclusion}

A novel mirowave coaxial cable sensor has been fabricated using a home made matel-ceramic coaxial cable Fabry-Pérot interferometer (MCCC-FPI). The sensor operates by monitoring its interferogram phase shifts caused by changes in the dielectric contant of the fluid flowing through the sample chamber in between the two reflectors. The MCCC-FPI sensor showed the capability of determining dielectric constants for fluids in high frequency range by a simple operation protocol that only requires a simple scan of the reference interferometric spectrum when the sensing chamber is filled with air or under vacuum to determine the actual inter-reflector chamber length. The sensor has been validated by dielectric constant measurements for single oils at room temperature and demonstrated for measurements at different temperatures. The sensor has also been successfully operated for continuous online monitoring of dielectric constant for the sesame oil-water mixture with changing compositions. The Bottcher's model of effective medium theory has been shown to be better suited for predicting the $\varepsilon_{r}$ of the highly molecularly asymmetric sesame oil-water mixture than the Maxwell-Garnett and Bruggeman equations of spherical solutions.

\section{Acknowledgements}

This research was financially supported by the USA Department of Energy, National

Energy Technology Laboratories under grant number DE-FE0022993.

\section{References}


[1] T. Friiso, T. Tjomsland, Monitoring of Density Changes in Low Permittivity Liquids by MicrowavePermittivity Measuerments with an Open-ended Probe, Meas. Sci. Technol. 8 (1997) 1295-1305.

[2] S.N. Jha, K. Narsaiah, A.L. Basediya, R. Sharma, P. Jaiswal, R. Kumar, R. Bhardwaj, Measurement Techniques and Application of Electrical Properties for Nondestructive Quality Evaluation of Foods - A Review. J. Food Sci. Technol. 48(2011) 387-411.

[3] M. T. Jilani, M. Z. Rehman, A. M. Khan, M. T. Khan, S. M. Ali, A Breif Review of Measuring Techniques for Characterizion of Dielectric Materials, Int. J. Inf. Technol. Electr. Eng. 1 (2012) 1-5.

[4] A. Göllei, A. Vass, E. Pallai, M. Gerzson, L. Ludányi, J. Mink, Apparatus and method to measure dielectric properties $\varepsilon$ ' and $\varepsilon ”$ of ionic liquids. Rev. Sci. Inst. 80 (2009) 044703-7.

[5] A. P. Gregory, R. N. Clarke, A Review of RF and Microwave Techniques for Dielectric Measurements on Polar Liquids, IEEE Trans. Dielectr. Electr. Insul. 13 (2006) 727-742.

[6] J. Huang, T. Wang, L. Hua, J. Fan, H. Xiao, L. Ming, A Coxial Cable Fabry-Perot Interferometer for Sensing Applications, Sensors 13 (2013) 15252-15260.

[7] T. Wei, S. Wu, J. Huang, H. Xiao, J. Fan, Coaxial Cable Bragg Gratings, Appl. Phys. Lett. 99 (2011) 113517.

[8] T. Wei, J. Fan, H. Xiao, Coaxial Cable Bragg Grating Assisted Microwave Coupler, Rev. Sci. Instrument. 85 (2014) 014703.

[9] A. Trontz, B. Cheng, S. Zeng, H. Xiao, J. Dong, Development of Metal-Ceramic Coaxial Cable Fabry-Pérot Interferometric Sensors for High Temperature Monitoring. Sensors 15 (2015) 24914-24925.

[10] J. Huang, X. Lan, W. Zhu, B. Cheng, J. Fan, Z. Zhou, H. Xiao, Interferogram Reconstruction of Cascaded Coaxial Cable Fabry-Perot Interferometers for Distributed Sensing Application, IEEE Sensors J. 16 (2016) 4495-4500. 
[11] T. Mathew, A. D. Vyas, D. Tripathi. Dielectric Properties of Some Edible and Medicinal Oils at Microwave Frequency. Can. J. Pure Appl. Sci. 3 (2009) 953-957.

[12] A. Cataldo, E. Piuzzi, G. Cannazza, E. De Bendetto, L. Tarricone, Quality and Anti-adulteration Control of Vegetable Oils through Microwave Dielectric Spectroscopy. Meas. 43 (2010) 1031-1039.

[13] N.E. Bengtsson, P. O. Risman, Dielectric Properties of Foods at $3 \mathrm{GHz}$ as Determined by a Cavity Perturbation Technique. J. Microw. Power (1971) 106-123.

[14] Q. Zhang, T. H. Jackson, A. Ungan, Numerical Modeling of Microwave Induced Natural Convection. Int. J. Heat Mass Transfer 43 (2000) 2141-2154.

[15] A. Carey, "The Dielectric Constant of Lubrication Oils", Computational Systems Incorporated 835 Innovation Drive Knoxville, TN 37932, pp. 675-683, 1998.

[16] T. Meissner, F. J. Wentz, The Complex Dielectric Constant of Pure and Sea Water From Microwave Satellite Observations. IEEE Trans. Geosci. Remote Sens. 42 (2004) 1836-1850.

[17] W. E. Pace, W.B. Westphal, S.A. Goldblith, Dielectric Properties of Commercial Cooking Oils, J. Food Sci. 33 (1968) 30-36.

[18] J. Lou, T.A. Hatton, P.F. Laibinis, Effective Dielectric Properties of Solvents Mixtures at Microwave Frequencies, J. Phys. Chem. A 101 (1997) 5265-8.

[19] Z. H. Shah, Q. A. Tahir, Dielectric Properties of Vegetable Oils. J. Sci. Res. 3 (2011) 481-492.

[20] W. Wen, H. Ma, W.Y. Tam, P. Sheng, Frequency and Water Content Dependencies of Electrorheological Properties, Phys. Rev. E 52 (1997) R1294-1297;

[21] J. Lou, T.A. Hatton, P.E. Laibinis, Effective Dielectric Properties of Solvent Mixtures at Microwave Frequencies, J. Phys. Chem. A 101 (1997) 5262-5268;

[22] C. J. F. Bottcher, Theory of Electric Polarization, Elsevier Scientific Publishing Company, Amsterdam, 1952. 